\title{
Gastric adenocarcinoma with choriocarcinomatous differentiation in the remnant stomach, 54 years after Billroth II partial gastrectomy
}

\author{
Mehmet Yildirim , Nazif Erkan¹, Enver Vardar², Enver Ilhan, Zehra Erkul² \\ 'Department of Surgery, Izmir Bozyaka Education and Research Hospital, Izmir, Turkey, ${ }^{2}$ Department of Pathology, Izmir \\ Bozyaka Education and Research Hospital, Izmir, Turkey
}

\begin{abstract}
The gastric remnant cancer is defined as a cancer that has developed 5 or more years after distal gastric resection for benign diseases of the stomach. The choriocarcinomatous differentiation of adenocarcinoma in the stomach is a very rare tumor. A 75-year-old woman underwent resection of remnant stomach which she had operated for benign gastric pathology 54 years ago. Pathologic examination of the resection revealed areas of choriocarcinomatous differentiation in addition to classical adenocarcinoma. In the literature on English language, this report presents the first case of adenocarcinoma associated with choriocarcinomatous differentiation in the gastric remnant. The treatment of these patients depends on the clinical setting, and early diagnosis is an important factor for the treatment strategies. Therefore, due to the probability of arising adenocarcinoma in the remnant stomach, the patient should be investigated with endoscopic examination periodically, despite the long postoperative period.
\end{abstract}

Keywords: Gastric cancer; choriocarcinoma

\section{INTRODUCTION}

The gastric remnant cancer (GRC) is defined as a carcinoma that arises in the stomach remnant following previous partial gastrectomy for benign diseases. The length of time after operation is important for the diagnosis of remnant cancer and it is usually defined as a cancer that has developed after 5 years of subtotal gastrectomy $(1,2)$. Adenocarcinomas with choriocarcinomatous differentiation are very

\footnotetext{
*Corresponding author: Mehmet Yildirim, Department of Surgery, Izmir Bozyaka Education and Research Hospital, Atakent Mah, Bergama 2 Apt,32/1, Bostanli, Izmir, Turkey, Tel.: 0090532 2855557. E-mail: mehmetyildi@gmail.com
}

Submitted: 24 April 2016/Accepted: 06 May 2016

DOI: http://dx.doi.org/10.17532/jhsci.2016.352 rare in the gastrointestinal tract. Pure choriocarcinomas or carcinomas associated with choriocarcinomatous areas are most commonly seen in the uterus, followed by the gonads, mediastinum, retroperitoneum, and rarely in the stomach and remnant stomach. Primary gastric choriocarcinoma was first reported by Davidson in 1905 and accounts for $0.08 \%$ of all primary gastric cancers (3). To our knowledge, there have been no published reports concerning the choriocarcinomatous differentiation of adenocarcinoma in the gastric remnant.

In this report we present a case of GRC with histology of adenocarcinoma with choriocarcinomatous differentiation in the gastric remnant after the patient had undergone a Billroth II subtotal gastrectomy 54 years earlier. 


\section{CASE REPORT}

A 75-year-old woman was admitted to our Surgical Department with vague epigastric pain. She had a history of a subtotal gastrectomy with Billroth II reconstruction for a benign condition approximately 54 years ago. She had no clinicopathological complaints and there was no endoscopic follow-up of upper gastrointestinal tract during this 54-year period. Laboratory findings were unremarkable. There was no evidence of gastric thickening or tumoral mass at computerized tomography. No pathological findings were found in the other intra-abdominal organ systems, including the genital system. Upper gastrointestinal endoscopy visualized an ulcerated polypoid lesion with the largest size of $0.6 \mathrm{~cm}$ at the anastomotic line (Figure 1) and the histological diagnosis of endoscopic biopsy was adenocarcinoma.

The patient underwent a total gastrectomy with Roux-en-Y esophagojejunostomy reconstruction as the gastric mass was found just above the anastomosis line extending to the subserosal layer. Macroscopic pathological examination revealed irregular tumor mass with ulcerative growth pattern largest size of $4 \mathrm{~cm}$ and multiple hemorrhagic and necrotic foci were seen at the anastomosis line (Figure 2). On the cut surface, the central of the tumor was hemorrhagic and necrotic and the peripheral zone of the tumor mass was whitish-gray colored. Microscopically, the tumor had two components (Figure 3A and 3B). The first component was a moderately differentiated adenocarcinoma, accounting for approximately $70 \%$ of the entire tumor sections and the second component had highly pleomorphic appearance and was accounting for approximately $30 \%$ of the entire tumor. There were highly pleomorphic cells associated with many syncytiotrophoblast-like cells with bizarre nuclei, especially in the hemorrhagic areas. Histological features of this component were consistent with choriocarcinoma. Immunohistochemically, syncytiotrophoblast-like cells and some highly pleomorphic cells were strongly positive for beta Human Chorionic Gonadotropin antibody ( $\beta-\mathrm{hCG}$ ) (Figure 4). However, there was no staining in the classical moderately differentiated adenocarcinomatous component for the $\beta$-hCG antibody.

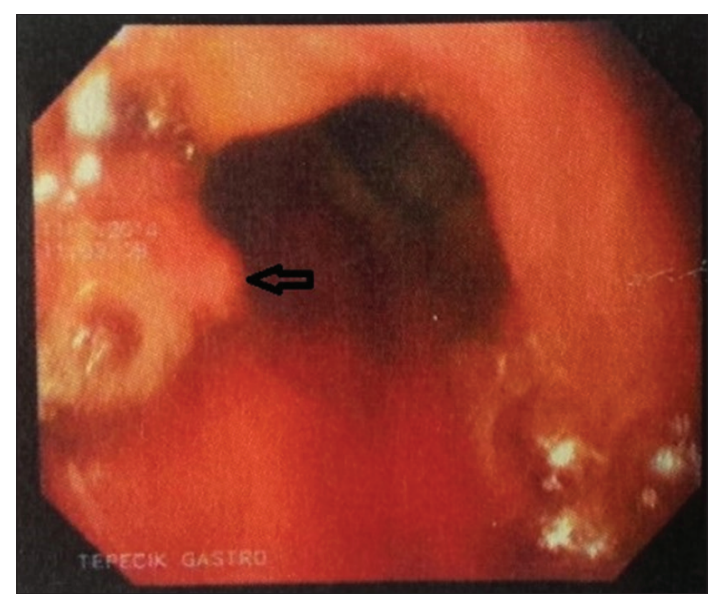

FIGURE 1. The endoscopic shape of the lesion shows a polypoid lesion with central ulceration (arrow).

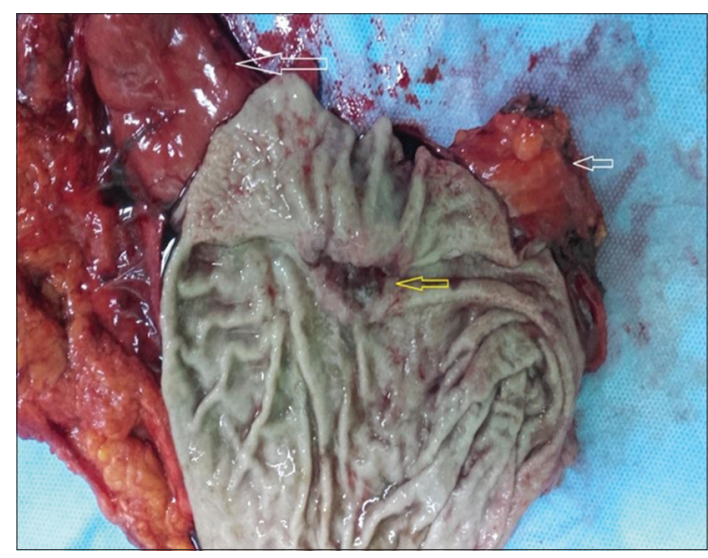

FIGURE 2. Macroscopic examination shows ulcerated tumor at the anastomosis line (large arrow: efferent loop; small arrow: afferent loop; yellow arrow: ulcer).

Immunohistochemically, neoplastic cells were diffusely positive for CK7 (cytokeratin) and pancytokeratin, focal mild positive for PLAP (Placental Alkaline Phosphatase) antibody and hPL (human placental lactogen), and negative for vimentin. Based on these histopathological findings, we diagnosed the lesion as gastric adenocarcinoma with choriocarcinomatous differentiation. Histopathologically, the tumor infiltrated the subserosal area and was classified as pathological tumor stage T3. There was no spread to the lymph nodes $(0 / 21)$ and there was no lymphovascular/perineural invasion. The patient was considered clinically stage IIA. Postoperative 


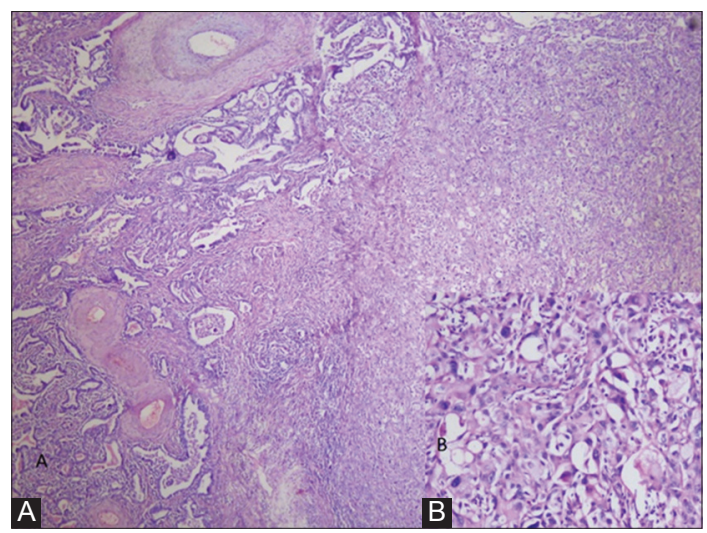

FIGURE 3. (A) Microscopically, the tumor had two components. The first component was a moderately differentiated adenocarcinoma (left side of the image) and the second component were solid areas (right side of the image). (B) Microscopic examination of the hemorrhagic areas showed highly pleomorphic tumor cells and many syncytiotrophoblast-like cells with bizarre nuclei.

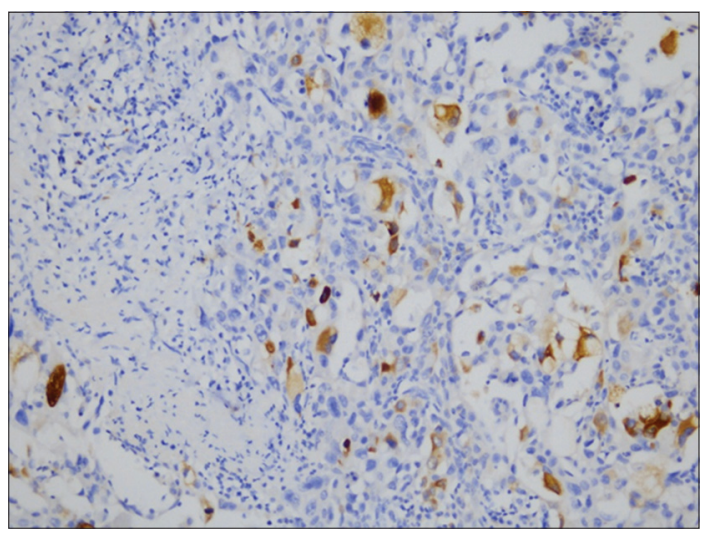

FIGURE 4. Immunohistochemically, the multinucleated choriocarcinomatous syncytiotrophoblast-like cells, stained with beta-Human Chorionic Gonadotropin antibody ( $\beta-h C G)$, were observed.

laboratory findings revealed the $\beta$-hCG level of $6.7 \mathrm{mIU} / \mathrm{ml}$ (normal level is under 5). After a year follow-up, there was no evidence for recurrent disease. Furthermore the $\beta$-hCG levels were within normal limits.

\section{DISCUSSION}

Cancer in the remnant stomach was classified into three types by Tanigawa, including a new developed cancer, remaining cancer at the primary operation (residual cancer), and recurrent cancer. Later,
Kaminishi described a newly cancer as a cancer developed more than 10 years after partial gastrectomy for benign or malignant disease (4). Also, a definition of a recurrent or residual cancer means that the cancer has developed less than 10 years after the surgery of the gastric malignancy. In the literature, the time between the operation and appearing GRC is between 5 and 40 years. According to this classification, our case was classified as a newly developed cancer in the remnant stomach. GRC is mostly observed around the gastroenterostomy line, and the pancreaticobiliary reflux may be the trigger for etiopathogenetic process of developing GRC. Because the clinical and laboratory findings of GRC are nonspecific at the early stage, these tumors are commonly presented at later stages. According to the Japanese Research Society of Gastric Cancer, nearly $30 \%$ of the newly developed cancers were found as differentiated adenocarcinoma. The majority of these tumors are adenocarcinomas, and the reports of classical adenocarcinoma associated with choriocarcinomatous differentiation are very rare.

A theory indicating a relationship with degeneration of a gastric teratoma has not been proven. The precise pathogenesis has not been clear, but several mechanisms including dedifferentiation of gastric adenocarcinoma, degeneration of gastric teratoma, or gonadal origin have been proposed. The choriocarcinomatous differentiation basically consists of cytotrophoblastic and syncytiotrophoblastic cells. Histopathological findings of the gastric biopsy are extremely elusive because small biopsy specimens are not adequate for accurate diagnosis. In this case, we found that the results were compatible with adenocarcinoma in endoscopic biopsy materials, and due to the scantiness of the biopsy materials choriocarcinomatous areas were not observed. Thus, larger and multiple gastric biopsy samples may be useful for definite diagnosis, preoperatively. Because elevated $\beta$-hCG levels in these patients are frequently observed, the level of $\beta$-hCG could be used to follow up and monitor the tumor recurrence in a patient, especially in post-operative period. However, mild elevation might be rarely seen in adenocarcinomas. In the presented case, the preoperative levels of serum $\beta$-hCG were not examined, because preoperative $\beta$-hCG levels are not examined in our routine clinical practice. In the post-operative period, 
$\beta$-hCG level of the patient was only mildly elevated. Some authors reported that the elevated $\beta$-hCG levels may be associated with poor prognosis (5).

In adenocarcinoma associated with choriocarcinomatous areas, surgical approach usually shows no difference from other classical gastric adenocarcinomas. Resection of the tumor mass with clear surgical margins on gastrectomy specimens are the best treatment modalities. Scars and fibrous adhesions due to previous operation may be additional difficulties in performing gastrectomy in the patient. On the other hand, in another study, resectability ratio was not different between the patients with GRC and native gastric cancer patients. The literature data emphasize the problem of increased lymphatic and hematogenous metastasis similar to primary testicular and uterine choriocarcinomas. Nakao et al. reported that approximately $70 \%$ of gastric choriocarcinomas metastasized to liver or lymph nodes at the time of diagnosis (3). However, in our patient, we did not observed distant metastasis. Overall survival was not found to be different between gastric remnant cancer and proximal gastric cancer for native adenocarcinomas $(1,6)$. Nevertheless, a small number of reported choriocarcinomatous differentiation examples is inadequate to estimate the accurate survival rate in order to compare with pure gastric choriocarcinomas.

Reviewing the literature, we observed that all patients with nonspecific symptoms required an upper gastrointestinal endoscopy at the first presentation, which was the case in our study as well. Our patient was an extremely uncommon case of primary remnant gastric adenocarcinoma with choriocarcinomatous differentiation. This case report emphasizes the importance of periodic endoscopic examination in patients with gastric resection.

\section{CONFLICT OF INTEREST}

The authors declare no conflict of interest.

\section{REFERENCES}

1. Costa-Pinho A, Pinto-de-Sousa J, Barbosa J, Costa-Maia J. Gastric stump cancer: more than just another proximal gastric cancer and demanding a more suitable TNM staging system. Biomed Res Int. 2013(2);781896. http://dx.doi.org/10.1155/2013/781896.

2. Sapmaz F, Basyigit S, Uzman M, Simsek G, Akkan T, Nazligul Y. A gastric stump cancer with unusual appearance. Journal of Health Sciences. 2015;5(3):104-6.

http://dx.doi.org/10.17532/jhsci.2016.289.

3. Nakao A, Sakagami K, Uda M, Mitsuoka S, Yamashita N, Ito H. Gastric carcinoma with predominant choriocarcinomatous component. Int J Clin Oncol. 1998;3(6):403-5.

http://dx.doi. org/10.1007/BF00539222.

4. Kaminishi M, Shimoyama S, Yamaguchi H, Yamada H, Ogawa T, Oohara T. Classification and carcinogenesis of gastric stump cancer. Gastroenterol Surg.1993;16:1253-65.

5. Lee SM, Kim KM, Ro JY. Variants of gastric carcinoma: Morphologic and theranostic importance. In: Lazăr D, editor. Gastric carcinoma: New insights into current management. Rijeka, Croatia: In Tech; 2013. p. 195-7. DOI: $10.5772 / 45896$.

6. Ikeguchi M, Kondou A, Shibata S, Yamashiro H, Tsujitani S, Maeta M, et al. Clinicopathologic differences between carcinoma in the gastric remnant stump after distal partial gastrectomy for benign gastroduodenal lesions and primary carcinoma in the upper third of the stomach. Cancer. 1994;73(1):15-21.

http://dx.doi.org/10.1002/1097-0142(19940101)73:1<15::AID-CNCR2820730105>3.0.CO;2-J. 